\title{
Placenta Accreta Syndrome- A Calamitous Diagnosis
}

\author{
Barkha Vats ${ }^{1}$, Sukriti Ghai ${ }^{1}$, Reena Yadav ${ }^{1}$, Kanika Chopra*1 \\ Assistant Professor, Lady Hardinge Medical College, New Delhi, India.
}

*Corresponding author: Kanika Chopra, Assistant Professor, Lady Hardinge Medical College, New Delhi, India

Received date: November 18, 2021; Accepted date: December 02, 2021; Published date: December 08, 2021

Citation: Barkha Vats, Sukriti Ghai, Reena Yadav, Kanika Chopra (2021). Placenta Accreta Syndrome- A Calamitous Diagnosisy. J. Women Health Care and Issues. 4(7); DOI:10.31579/2642-9756/087

Copyright: (C) 2021 Kanika Chopra, This is an open access article distributed under the Creative Commons Attribution License, which permits unrestricted use, distribution, and reproduction in any medium, provided the original work is properly cited.

\begin{abstract}
:
Increasing incidence of Placenta accrete syndrome has become a worrisome issue due to its associated lifethreatening complications for both the mother and the fetus. The ideal management for PAS disorder remains the matter of debate still. The critical step in its effective management being its suspicion knowing the underlying risk factors and its diagnosis in antenatal period. Still, cesarean hysterectomy remains the gold standard procedure with many newer conservative approaches under evaluation. Our basic aim behind writing this review is to highlight the recent changes in classifying and diagnosing PAS owing to the ever-increasing incidence of this catastrophic entity. Also, it will emphasize the well-established role of radical over conservative management and also all modalities used in conservative management so far.
\end{abstract}

Keywords: placenta accreta syndrome; hysterectomy; pelvic devascularization; conservative approach

\section{Introduction}

Placenta accreta syndrome previously known as morbidly adherent placenta is a significantly grave obstetrical condition. PAS includes both abnormally adherent placenta where implantation of villi are in direct contact with myometrium without obvious plane of separation and abnormally invasive placenta where villi invade into the myometrium and even nearby organs. FIGO define PAS as grade 1, 2 and 3 [1]. It can be considered as a multifactorial pathology and results due to factors like defective decidua, abnormality in trophoblastic invasion, abnormal angiogenesis and thus neovascularization [2]. There has been a tremendous increase in the incidence of PAS in last three to four decades. The cited incidence of placenta accreta was 1 in 2510 in the 1980 which has risen to 1 to 533 in recent years. [3] The root cause of this rising incidence being increase in cesarean section rate. It accounts for considerable maternal mortality and morbidity and thus represent a major challenge to the obstetricians. So, it is prudent that having knowledge of risk factors, a high clinical suspicion is made, so that antenatal radiological diagnosis is made and patient managed in equipped facility. The standard treatment in majority of the cases of PAS is peripartum hysterectomy including our hospital, the exclusion being a case of focal accreta. Recently there was increasing inclination towards conservative approach, but radical treatment remains the best. Our basic aim behind writing this review is to highlight the recent changes in classifying and diagnosing PAS owing to the ever-increasing incidence of this catastrophic entity. Also, it will emphasize the well-established role of radical over conservative management and also all modalities used in conservative management so far.

\section{Identification of risk factors}

- Previous history of caesarean section- The astonishing increase in the incidence of PAS is attributed to the increasing incidence of caesarian sections in last few decades. According to a systemic review, the rate of PAS has increased from $0.3 \%$ in women with one previous caesarean delivery to $6.74 \%$ for women with five or more cesarean deliveries. [4]

- Placenta previa- For women with placenta previa, the risk of PAS is $3 \%, 11 \%, 40 \%, 61 \%$, and $67 \%$ for the first, second, third, fourth and fifth or more cesarean respectively. [5]

- Previous history of PAS

- Advanced maternal age

- Multiparity

- Prior uterine surgeries like myomectomy or curettage

- Manual removal of placenta

- Post-partum endometritis

- Cesarean scar pregnancy

- Artificial reproductive technology-Elevated serum estrogen levels at the time of embryo implantation have been proposed to be the cause of PAS. [5]

- Uterine pathology for example fibroid, bicornuate uterus, Asherman's syndrome

- Abnormal increase in placental biomarkers like MSAFP and beta HCG although not clinically useful, is a risk factor for PAS. Further studies are needed to identify the usefulness of serological markers along with ultrasonography. 


\section{Diagnosis}

Ultrasound is the first line diagnostic modality, but the signs seen and the terminology used happen to have variable heterogeneity. Moreover, diagnosis depends on the operator's experience. 3-D power doppler use is under investigation and may prove to improve antenatal diagnosis. MRI do have a role in cases like posterior placenta, ultrasound suggestive of parametrium invasion of placenta and in patients with high BMI. This imaging modality has a high sensitivity and specificity with disadvantage of being not available readily and being expensive.

\section{Management}

Management in a case of PAS is cesarean hysterectomy with the placenta in situ as the gold standard procedure as seen in figure 1, except in few cases of focally adherent placenta.

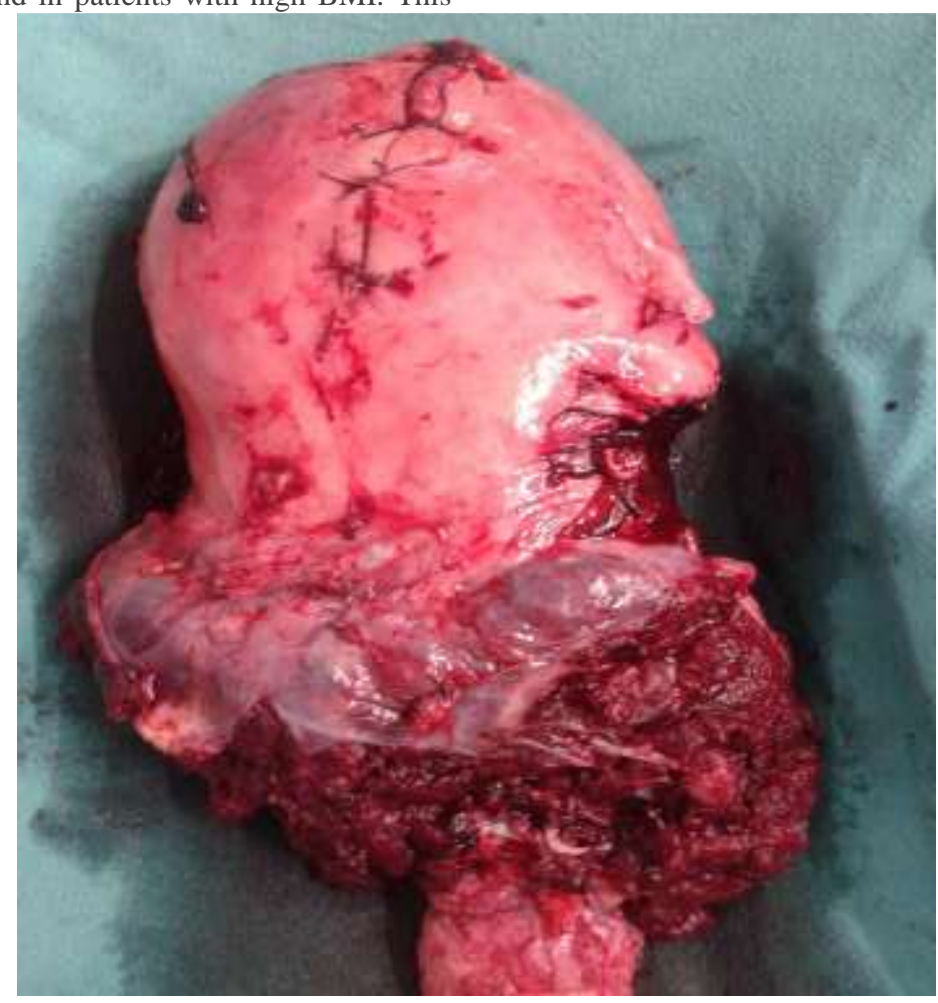

Figure 1: A post-operated specimen of Placenta Accreta syndrome operated in our department, FIGO grade 2.

IS-AIP defines focal adherence as $<50 \%$ of adherence or invasion involving anterior surface of the uterus. In these cases, resection of adherent placenta with the underlying myometrium can be done followed by reconstruction of uterine wall. Triple $\mathrm{P}$ procedure is another novel approach. It includes: a) Preoperative localization of placenta using ultrasound followed by delivery of fetus by transverse incision two finger breadth above it. b) Pelvic devascularization by inflating internal artery arteries balloons. c) Placental non separation with myometrial excision followed by reconstruction of the uterine wall.

Cases antenatally diagnosed should be referred or managed in a tertiary care center with a dedicated multidisciplinary team for optimum management of PAS. This team should include experienced obstetrician, pelvic surgeons, general surgeons, urologists, interventional radiologist, anesthesiologist, blood bank officer and strong nursing leadership. Delivery is planned at $35+0$ to $36+6$ if the risk factors for preterm delivery like preeclampsia, preterm rupture of membranes are not present. [6]

Alternative to conventional hysterectomy especially if there is bladder involvement is retrograde hysterectomy. In this approach retroperitoneal space is developed followed by internal iliac ligation. The vaginal vault is then opened posteriorly and a plane is developed through lateral dissection to separate the bladder. The blood loss in this procedure is lesser as compared to conventional hysterectomy. [7]

Delayed hysterectomy is another alternative. It is found to be beneficial because of prevention of complications during immediate hysterectomy like decrease risk of hemorrhage and need for massive blood transfusion.
In a large case series in which 13 women with placenta percreta underwent delayed hysterectomy after a median 41 days of elective cesarean section. It was observed that blood loss was markedly less as compared to cesarean hysterectomy. [8]

Endovascular intervention like balloon catheter, arterial embolization or combination of these two have been found to decrease blood loss in some case reports but are not recommended. ${ }^{9}{ }^{10}$ But these procedures have been found to be ineffective as seen in our hospital as well. The main reason behind this lies in the pathophysiology of development of PAS i.e., neovascularization and development of collaterals. There can be vessel injury, ischemia and thromboembolism related to these procedures.

Multitudinous other approaches have been defined in terms of number of case reports, series and retrospective studies highlighting the benefits of conservative approach, but none have been proved of much significance. The indications of conservative methods are also limited and includes:

- When intra-operative findings are suggestive of high risk of massive hemorrhage, injury to adjacent organs and thus high risk of morbidity or mortality in case if hysterectomy is proceeded.

- Women desirous of preserving fertility, after understanding the need for long term follow up.

The most important inception for conservative approach being no excessive bleeding following delivery of baby and patient hemodynamically stable with no coagulopathy. Informed consents prior to proceeding with it includes high risk of bleeding, infection, 
coagulopathy, uterine necrosis, need for hysterectomy in emergency, need for long term follow-up and risk of PAS in future pregnancies as well. Studies have shown that $31.4 \%$ cases of patients undergoing expectant management underwent hysterectomy due to hemorrhage [11]. In the largest case series done on the expectant management of PAS cases, highest success rate of $78.4 \%$ was found. Around $51.5 \%$ cases developed secondary postpartum hemorrhage and among these $44.4 \%$ underwent hysterectomy. [12]

The various methods used so far included use of balloon tamponade or compression sutures, stepwise uterine devascularization just ending before hysterectomy, uterine artery embolization or use of methotrexate after leaving the placenta in situ among many others.

Use of methotrexate is not recommended. It acts upon rapidly dividing cells and division of third trimester placental cells is limited. Also, methotrexate is responsible for maternal hematological and renal toxicities and it is contraindicated in breast feeding. [13,14]

Cervix as a natural tamponade technique is another conservative procedure in which anterior lip of the cervix is stitched with anterior uterine wall and posterior lip is stitched to posterior wall. A prospective study conducted on 40 women with diagnosed placenta accreta were managed by this technique. This was successful in controlling bleeding in 38 women. [15]

Hysteroscopic resection of retained placenta which can be done in one or two attempts. This procedure is not recommended for increta and percreta. A study was conducted on 12 women with persistent placental tissue, 2 with complete and 10 with partial retention of placenta, who underwent hysteroscopic resection. Only one of the women required hysterectomy in this study after incomplete removal after first sitting. Half of the women required more than one attempts. [16]

High intensity focused ultrasonography has been used but its safety needs to be checked through larger trials. Routine use of hysteroscopic resection with or without HIFU is not recommended due to side effects like uterine perforation and need for repeat procedure. [17]

In spite of increasing efforts to device new strategies for conservative management of cases of PAS, the role of cesarean hysterectomy cannot be undermined in many years to follow. Considering the high rates of morbidity of $40-50 \%$ and mortality in around $7 \%$ cases of PAS, all focus should not be just on the ways to reduce the risks associated with this havoc diagnosis but to decrease the cesarean section rates as the preventive strategy. It has to start at the institution level by closely auditing all indications of induction of labour and so forth cesarean sections being done on monthly basis. The indications of cesarean should be discussed in audit meetings so that any unnecessary cesarean if done can be prevented in future. Authors think one cesarean section prevented, may save one life. Cesarean audit is being done in our set-up as well with all attempts to decrease cesarean section rates.

\section{Conclusion}

It is one of the most dreaded complication as there is increased risk of post-partum hemorrhage, massive blood transfusion, peripartum hysterectomy, surgical injuries, ICU stay, sepsis, deep venous thrombosis, transfusion related lung injury, disseminated intravascular coagulation and even maternal death. Keeping all these factors in mind, measures should be taken to reduce the incidence of PAS and to diagnose it prenatally. Prevention strategies may include avoiding caesarean section birth, correct surgical techniques while closing the uterine incision, avoiding vigorous curettage, and treatment of postpartum endometritis. The most important contributor to the increased incidence is the rising cesarean section births. We know that cesarean section has revolutionized the fetal and maternity care but it must be performed only when indicated and should not be used as a surgery of convenience. Efforts should be made to promote vaginal delivery and reduce the cesarean rates.

\section{Conflict of interest: None}

\section{Acknowledgement: None}

\section{References}

1. Jauniaux E, Ayres de Campos D, Langhoff Roos J et al. FIGO classification for the clinical diagnosis of placenta accreta spectrum disorders. International Journal of Gynaecology and obstetrics.2019 Jul;146(1):20-24.

2. Biberoglu E, Kirbas A, Daglar K et al. Serum angiogenic profile in abnormal placentation. The journal of Maternal-Fetal and Neonatal Medicine. 2016 Oct 1;29(19):3193-3197.

3. Kyozuka H, Yamaguchi A, Suzuki D et al. Risk factor for placenta accreta spectrum: findings from the Japan environment and Children's study. BMC pregnancy and childbirth. 2019 Dec;19(1):1-7.

4. Cahill AG, Beigi R, Heine RP et al. Placenta accreta spectrum. American journal of obstetrics and gynecology. 2018 Dec 1;219(6): B2-16.

5. Modest AM, Toth TL, Johnson KM et al. Placenta Accreta Spectrum: in Vitro fertilization and Non-In Vitro fertilization and Placenta Accreta Spectrum in a Massachusetts Cohort. AM J Perinatol. 2020;5: s-0040-1713887.

6. Jauniaux E, Kingdom J, Silver RM et al. A comparison of recent guidelines in the diagnosis and management of placenta accreta syndrome disorders. Best practice and Research Clinical Obstetrics and Gynecology. 2020 Jun 27.

7. Selman AE. Caesarean hysterectomy for placenta previa/accreta using an approach via the, pouch of, Douglas. BJOG: An International, Journal of, Obstetrics and Gynecology. 2016 Apr;123(5):815-819.

8. Zuckerwise LC, Craig AM, Newton JM et al. Outcome following a clinical algorithm allowing for delayed hysterectomy in the management of severe placenta accreta spectrum. American Journal of Obstetrics and Gynecology. 2020 Feb 1;222(2):179-e1.

9. Dilauro MD, Dason S, Athreya S. Prophylactic balloon occlusion of internal iliac arteries in women with placenta accreta: literature review and analysis. Clinical radiology. 2012 Jun 1; 67(6):515520.

10. Bodner LJ, Nosher JL, Gribbin C et al. Balloon - assisted occlusion of the internal iliac arteries in patient with placenta accreta/percreta. Cardiovascular and interventional radiology. 2006 Jun;29(3):354-361.

11. Marcellin L, Delorme P, Bonnet MP et al. Placenta percreta is associated with more frequent severe maternal morbidity than placenta accreta. Am J Obstet Gynecol, 2018;219(2):193. E1193.e9.

12. Sentilhes L, Ambroselli C, Kayem G et al. Maternal outcome after conservative treatment of placenta accreta. Obstet Gynecol, 2010;115:526-534.

13. Fox KA, Shamshirsaz AA, Carusi D et al. Conservative management of morbidly adherent placenta: expert review. Am J Obstet Gynecol 2015; 213: 755-760.

14. Sentilhes L, Ambroselli C, Kayem G et al. Maternal outcome after conservative treatment of placenta accreta. Obstet Gynecol 2010; 115:526-534.

15. El Gelany SA, Abdelraheim AR, Mo 'men MM et al. The cervix as a natural tamponade in postpartum hemorrhage caused by placenta previa and placenta previa accreta: a prospective study. BMC pregnancy and childbirth. 2015 Dec;15(1):1-7. 
16. Legendre G, Zoulovits FJ, Kinn J et al. Conservative management of placenta accreta: hysteroscopic resection of retained tissues. J Minim Invasive Gynecol 2014; 21: 910-913.
17. Ye M, Yin Z, Xue M et al. High intensity focused ultrasound combined with hysteroscopic resection for the treatment of placenta accreta. BJOG 2017; 124 (suppl 3): 71-77.
This work is licensed under Creative

Commons Attribution 4.0 License

To Submit Your Article Click Here:

Submit Manuscript

DOI: $10.31579 / 2637-8892 / 149$
Ready to submit your research? Choose Auctores and benefit from:

$>$ fast, convenient online submission

$>$ rigorous peer review by experienced research in your field

$>$ rapid publication on acceptance

$>$ authors retain copyrights

$>$ unique DOI for all articles

$>$ immediate, unrestricted online access

At Auctores, research is always in progress.

Learn more https://auctoresonline.org/journals/psychology-and-mental-healthcare 